\title{
(6) OPEN ACCESS \\ Association between VEGF-A and VEGFR-2 polymorphisms and response to treatment of neovascular AMD with anti-VEGF agents: a meta-analysis
}

\author{
Mingxing $\mathrm{Wu}^{1}{ }^{1}$ Haibo Xiong, ${ }^{1,2}$ Yan $\mathrm{Xu}^{1}{ }^{1}$ Xiaojing Xiong, ${ }^{1}$ Hongmi Zou, \\ Minming Zheng, ${ }^{1}$ Xiuqing Wang, ${ }^{1}$ Xiyuan Zhou ${ }^{1}$
}

\begin{abstract}
- Additional material is published online only. To view please visit the journal online (http://dx.doi.org/10.1136/ bjophthalmol-2016-309418).

'Department of Ophthalmology, The Second Affiliated Hospital of Chongqing Medical University, Chongqing, China

2Department of Ophthalmology, Chongqing General Hospital, Chongqing, China
\end{abstract}

Correspondence to Dr Xiyuan Zhou, 74\#Linjiang Road, Chongqing, China; zhouxiyuan2002@aliyun.com

MW and HX contributed equally.

Received 25 July 2016 Revised 19 September 2016 Accepted 28 September 2016 Published Online First 21 October 2016

CrossMark

To cite: Wu M, Xiong $\mathrm{H}$, Xu Y, et al. Br J Ophthalmol 2017;101:976-984.

\section{ABSTRACT}

Aims The purpose of this study is to investigate whether gene polymorphisms of the vascular endothelial growth factor A (VEGF-A) and its receptor (VEGFR-2) have a pharmacogenetics effect on the anti-VEGF treatment for neovascular age-related macular degeneration (nAMD).

Methods We carried out a meta-analysis focusing on the relationship between VEGF-related gene polymorphisms and treatment response of nAMD.

Results For the single nucleotide polymorphisms (SNPS) within VEGF-A and VEGFR-2, anti-VEGF treatment was much more effective in patients with nAMD having rs 833061 (CC vs TT:OR=2.222, 95\% Cl 1.252 to 3.944, $p=0.006$; $C T$ vs TT: $O R=2.537,95 \% \mathrm{Cl} 1.478$ to 4.356, $\mathrm{p}=0.001$ and CC vs CT+TT: $\mathrm{OR}=2.362,95 \% \mathrm{Cl}$ 1.414 to $3.946, p=0.001$ ), particularly for Asians (CC vs TT: $O R=2.903,95 \% \mathrm{Cl} 1.150$ to 7.330, $p=0.024$; CT vs TT: $O R=3.849,95 \% \mathrm{Cl} 1.522$ to $9.733, p=0.004$ and CC vs CT+TT: OR=3.339, $95 \% \mathrm{Cl} 1.369$ to 8.145 , $p=0.008$, respectively). In subgroup analysis, rs833061 was more likely to be a predictor of response to antiVEGF therapy specifically when ranibizumab (RBZ) only regime was adopted or visual acuity (VA) was taken as the standardised assessment of outcome. No association with response to anti-VEGF treatment was detected for the other eight polymorphisms.

Conclusions Pharmacogenetics of VEGF-A

polymorphism rs833061 may play a positive role in response to anti-VEGF therapy for nAMD.

Age-related macular degeneration (AMD) is the leading cause of irreversible blindness in people aged over 50 in the developed world. There are two types of AMD, the non-exudative (dry or atropic) AMD and the exudative (wet or neovascular) AMD. The later form, neovascular AMD (nAMD), is characterised by the presence of choroidal neovascularisation beneath the fovea. It has been demonstrated that vascular endothelial growth factor (VEGF), a signal protein, plays a key role in the regulation of angiogenesis, vascular leakage and inflammation that is characteristic of nAMD by stimulating growth of new blood vessels. ${ }^{1}$ Intravitreal injections of anti-VEGF agents, such as the monoclonal antibody fragment ranibizumab (RBZ, Lucentis, Genentech, San Francisco, California, USA), the monoclonal antibody bevacizumab (BVZ, Avastin, Genentech, San Francisco, California, USA) and the aflibercept, are currently considered part of the standard treatment regimen for neovascular AMD (nAMD). ${ }^{2}$ Results from the Comparison of AMD Treatments Trials (CATTs) and other multicentre clinical trials that compared RBZ and BVZ indicate that both drugs provide dramatic and lasting visual improvements in patients $^{3-5}$ (for a lack of efficient clinical information for aflibercept, here we emphasise on the RBZ and BVZ). However, there is individual variation in the initial response to therapy and in the durability of the clinical effect. One logical explanation for the variability in treatment response might be differences in genetic background. It is well established that several genetic risk variants are associated with the development and progression of AMD. ${ }^{6}$ Recent researches on outcome determinants have focused on the role of these variants on the response to anti-VEGF therapy with inconsistent results. ${ }^{7}$

An obvious next strategy to identify pharmacogenetic markers that may predict antiangiogenic therapy is to analyse polymorphisms in genes within the VEGF signalling pathway. The family of VEGF genes consists of six members, five structurally related (VEGF-A, VEGF-B, VEGF-C, VEGF-D and placental growth factor $)^{8}$ and the orf-virus-encoded VEGF-E. ${ }^{9}$ The protein products of these genes bind to one of three VEGF receptors (VEGFR-1, VEGFR-2 and VEGFR-3) in order to elicit a cellular response. ${ }^{8}$ Substantial evidence indicated that VEGF-A is a major mediator of angiogenesis and vascular leakage in nAMD. Various VEGF inhibitors have been clinically developed. Among these, RBZ is a high affinity recombinant Fab that neutralises all isoforms of VEGF-A. ${ }^{9}$ The success of such therapies resulted in several studies of VEGF-A single nucleotide polymorphisms (SNPs) as prognostic indicators of AMD pathogenesis. They were evaluated by a recent meta-analysis that demonstrated that SNPs within VEGF-A (the presence of a C-nucleotide in rs833061 or TT in rs1413711) may be associated with AMD pathogenesis. ${ }^{10}$ Given that VEGF SNPs are involved in disease pathogenesis and that the highly successful anti-VEGF nAMD therapies target VEGF-A, SNPs within VEGF-A were considered to be the most likely to predict how a patient with nAMD will respond to such therapies. Meanwhile, as VEGF-A binds to VEGFR-2 to stimulate angiogenesis within tissues, it is plausible that SNPs within VEGFR-2 could predict response to anti-VEGF therapy. 
Thus far, several association studies regarding the predictive role of polymorphisms within VEGF-A and VEGFR-2 have been reported, though the results were inconclusive yet. For example, for polymorphism rs699947 in VEGF-A, patients carrying AA genotype revealed an absence of early functional response to $\mathrm{RBZ}^{11}$ an increased chance of good response in another, ${ }^{12}$ while a third study concluded that there was no association between visual acuity (VA) outcome and this genotype. ${ }^{13}$ Therefore, we carried out a meta-analysis focusing on the relationship between VEGF-related gene polymorphisms and treatment response of nAMD in order to obtain a more convincing and precise conclusion. To the best of our knowledge; this is the first meta-analysis focusing on the relationship between VEGF-A and VEGFR-2 polymorphisms and response to treatment of nAMD with anti-VEGF agents.

\section{METHODS}

\section{Search strategy}

We conducted searches of PubMed and EMBASE, using the terms ('vascular endothelial growth factor' or 'VEGF') and ('age-related macular degeneration' or 'AMD'). A manual search was performed by checking the reference lists of original reports and review articles to identify studies not yet included in the computerised databases. The final search was carried out on 29 January 2016, without restrictions regarding publication year or language.

\section{Inclusion and exclusion criteria}

Articles were considered eligible for inclusion in the meta-analysis if the studies met the following inclusion criteria: (1) articles evaluating the relationship between the VEGF-related gene polymorphisms and response to anti-VEGF treatment for nAMD; (2) independent retrospective or prospective association study (3) with sufficient available data to estimate an OR with 95\% CI. Exclusion criteria were as follows: (1) duplication of previous publications; (2) comment, review and editorial; (3) family-based studies of pedigrees and (4) study with no detailed genotype data. When there were multiple publications from the same population, only the largest study was included. Study selection was achieved by two investigators independently, according to the inclusion and exclusion criteria by screening the title, abstract and full text. Any dispute was solved by discussion.

\section{Data extraction}

The data were extracted independently by two reviewers (MW and HX). The following contents were collected: category of genes and polymorphisms, name of first author, year of publication, study design, location and ethnicity of the trial, treatment modality, number of subjects, mean age and female ratio of the patients, duration of the study, definition of a good response and genotype distributions. Two authors checked the extracted data and reached consensus on all the data. If a dissent existed, they would recheck the original data of the included studies and have a discussion to reach consensus. If the dissent still existed, the third investigator would be involved to adjudicate the disagreements $(\mathrm{Xu})$.

\section{Quality assessment}

We adopted the Newcastle Ottawa Scale (NOS) (accessed via http://www.ohri.ca/programs/clinical_epidemiology/oxford.asp) to evaluate the quality of included studies by a modified checklist, in which a study was judged on three categories: selection (four items, one star each), comparability (one item, up to two stars) and exposure/outcome (three items, one star each). A 9-point scale of the NOS (range, 0-9 points) has been developed for the evaluation. Studies were defined as high quality if they had more than 7 points; they were defined as medium quality if they had between 4 and 6 points and as poor quality if they had fewer than 4 points. Studies with NOS score above 4 points were included in the final analysis. Any disagreement was adjudicated by a third author.

\section{Statistical analysis}

The quantitative data were entered into Cochrane Review Manager (Stata12.0 software, StataCorp, College Station, Texas, USA). The strength of association was evaluated using the summary ORs and 95\% CIs of each gene polymorphism. A p value $<0.05$ was considered statistically significant on the test for overall effect. Heterogeneity was tested by the Q-statistic and the $\mathrm{I}^{2}$ value. ${ }^{14}$ The Q-statistic was considered significant when $\mathrm{p}<0.10$. The $\mathrm{I}^{2}$ values indicated no $(0 \%-24.9 \%)$, low (25\%-49.9\%), moderate $(50 \%-74.9 \%)$ or high $(75 \%-100 \%)$ interstudy heterogeneity. ${ }^{14} 15$ If the $\mathrm{p}$ value for the Q-statistic was $<0.10$ or the $\mathrm{I}^{2}$ value $\geq 50 \%$, a random-effects model was used, otherwise a fixed-effects model was adopted. ${ }^{16}$ The genetic association was assessed using different genetic models, including allelic ( $\mathrm{A}$ vs $\mathrm{B})$, dominant $(\mathrm{AA}+\mathrm{AB}$ vs $\mathrm{BB})$, recessive (AA vs $\mathrm{AB}+\mathrm{BB}$ ) and codominant (homozygous: $\mathrm{AA}$ vs $\mathrm{BB}$; heterozygous: AA vs $\mathrm{AB}$ ) models. The most appropriate genetic model was chosen for further analyse. We also performed subgroup analysis by ethnicity, treatment and definition of a good response. Begger's test and Egger's test were employed to quantitatively assess publication bias $(p<0.05$ was considered representative of significant statistical publication bias). ${ }^{17} 18$

\section{RESULTS}

\section{Overall characteristics of selected studies and quality}

\section{assessment}

In the literature search, a total of 1228 records, published between 1 May 1989 and 29 January 2016 were retrieved from the PubMed, EMBASE and Web of Science database. Among them, 734 were duplicated records. From the remaining 494 articles, we found 16 full-text articles assessed for eligibility, then excluded five papers with incomplete data (although we have contacted the authors via email or telephone, unfortunately we still could not get available data), 2 with duplicated data and 1 review. Finally, eight studies investigating totally nine genetic variations in VEGF-related genes were included in this meta-analysis. ${ }^{11-13}{ }^{19-23}$ Figure 1 provides a flow diagram of the search procedure and results. Regarding ethnicity, seven studies were included, mostly Caucasians, only one study included East Asians. According to the NOS, which was used for quality assessment, all of the studies included had high quality scores of 7 or 8 (table 1). The average score for all eight studies included in the meta-analysis was 7.625. The majority (five out of eight) of the studies used RBZ only, while two studies used either RBZ or BVZ and one study used BVZ only. In our study, the follow-up period ranged from 4 to 12 months and we defined a good response mainly based on two improvement criteria in all participating patients as follows: (1) Best-corrected VA (BCVA) improvement: we considered improvement when the patients gained more than five letters in the ETDRS chart compared with BCVA at baseline or when patients' loss of VA $<30 \%$ of letters; (2) optical coherence tomography (OCT) improvement: improvement of central subfield retinal thickness as measured by OCT compared with the baseline OCT thickness. In the limited number of articles included in this meta-analysis, one 
Figure 1 Flow diagram of studies included in this meta-analysis.

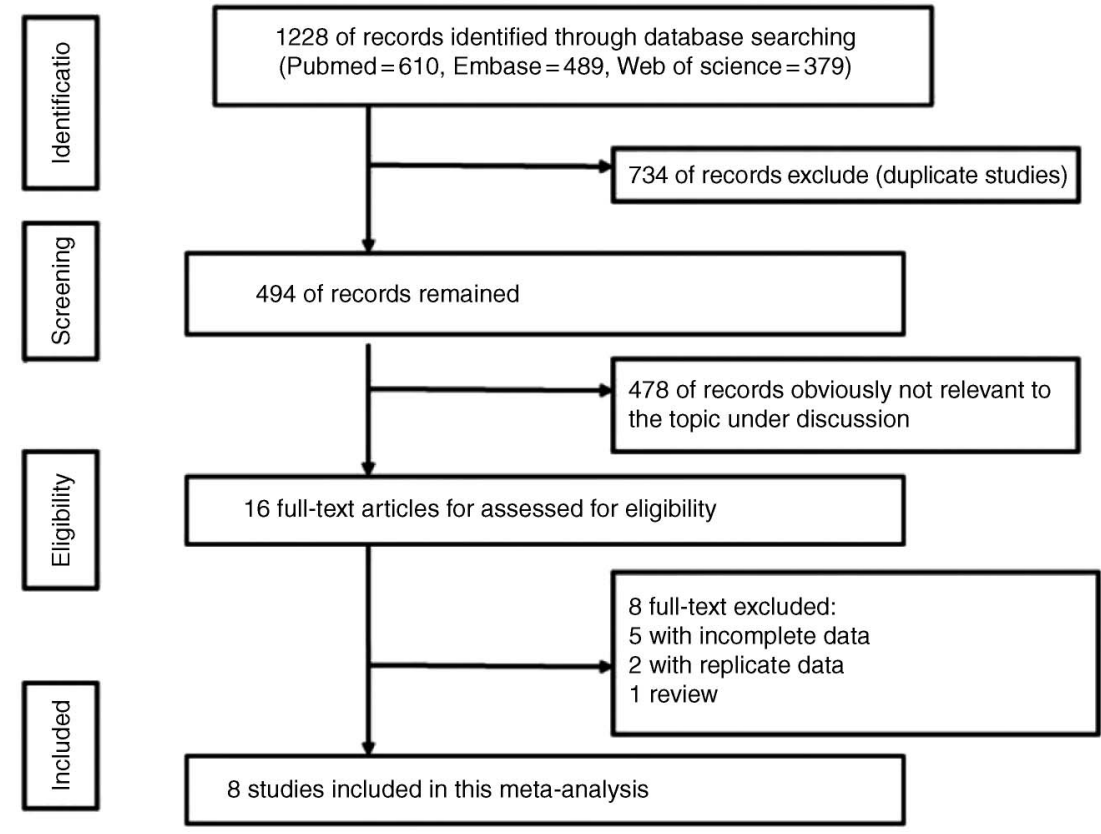

Table 1 Characteristics of the studies included and quality scores in the current meta-analysis

\begin{tabular}{|c|c|c|c|c|c|c|c|c|c|c|c|}
\hline Gene & Polymorphism & $\begin{array}{l}\text { Study group } \\
\text { (year) }\end{array}$ & Design & Location & Ethnicity & Treatment & $\begin{array}{l}\text { No. of } \\
\text { patients }\end{array}$ & $\begin{array}{l}\text { Mean age } \\
\text { (years) }\end{array}$ & $\begin{array}{l}\text { Female } \\
(\%)\end{array}$ & $\begin{array}{l}\text { Follow-up } \\
\text { (months) }\end{array}$ & $\begin{array}{l}\text { Quality } \\
\text { score }\end{array}$ \\
\hline \multirow[t]{22}{*}{ VEGF-A } & $\begin{array}{l}\text { Rs699947 } \\
A>C\end{array}$ & $\begin{array}{l}\text { Hautam'a'ki } \\
(2013)\end{array}$ & Prospective & Finland & Caucasian & BVZ & 96 & 78.0 & 63.5 & 4 & 8 \\
\hline & & Kitchens (2013) & Retrospective & USA & Caucasian & $\begin{array}{l}\text { RBZ or } \\
\text { BVZ }\end{array}$ & 99 & 80.0 & 67.3 & 4 & 8 \\
\hline & & Lazzeri (2013) & Prospective & Italy & Caucasian & RBZ & 64 & 76.3 & 45.3 & 9 & 7 \\
\hline & & Park (2014) & Prospective & South Korea & East Asia & RBZ & 270 & 69.5 & 43.6 & 5 & 8 \\
\hline & & Hagstrom (2014) & Prospective & USA & Caucasian & $\begin{array}{l}\text { RBZ or } \\
\text { BVZ }\end{array}$ & 835 & 78.5 & 61.2 & 12 & 8 \\
\hline & & $\begin{array}{l}\text { Cruz-Gonzalez } \\
(2014)\end{array}$ & Prospective & Spain & Caucasian & $\mathrm{RBZ}$ & 74 & 71.6 & 52.1 & 12 & 8 \\
\hline & & $\begin{array}{l}\text { Van Asten } \\
(2014)\end{array}$ & Prospective & $\begin{array}{l}\text { The Netherlands, } \\
\text { Germany, Canada }\end{array}$ & Caucasian & $\mathrm{RBZ}$ & 375 & $\mathrm{~N} / \mathrm{A}$ & 56.2 & 3 & 7 \\
\hline & Rs 699946 & Park (2014) & Prospective & South Korea & East Asia & RBZ & 273 & 69.5 & 43.6 & 5 & 8 \\
\hline & $A>G$ & Hagstrom (2014) & Prospective & USA & Caucasian & $\begin{array}{l}\text { RBZ or } \\
\text { BVZ }\end{array}$ & 835 & 78.5 & 61.2 & 12 & 8 \\
\hline & $\begin{array}{l}\text { Rs } 833069 \\
\text { C>T }\end{array}$ & Hagstrom (2014) & Prospective & USA & Caucasian & $\begin{array}{l}\text { RBZ or } \\
\text { BVZ }\end{array}$ & 835 & 78.5 & 61.2 & 12 & 8 \\
\hline & & $\begin{array}{l}\text { Van Asten } \\
\text { (2014) }\end{array}$ & Prospective & $\begin{array}{l}\text { The Netherlands, } \\
\text { Germany, Canada }\end{array}$ & Caucasian & $\mathrm{RBZ}$ & 368 & $\mathrm{~N} / \mathrm{A}$ & 56.2 & 3 & 7 \\
\hline & $\begin{array}{l}\text { Rs } 833061 \\
\text { C>T }\end{array}$ & Kitchens (2013) & Retrospective & USA & Caucasian & $\begin{array}{l}\text { RBZ or } \\
\text { BVZ }\end{array}$ & 99 & 80.0 & 67.3 & 4 & 8 \\
\hline & & Park (2014) & Prospective & South Korea & East Asia & RBZ & 271 & 69.5 & 43.6 & 5 & 8 \\
\hline & & $\begin{array}{l}\text { Cruz-Gonzalez } \\
(2014)\end{array}$ & Prospective & Spain & Caucasian & $\mathrm{RBZ}$ & 74 & 71.6 & 52.1 & 12 & 8 \\
\hline & $\begin{array}{l}\text { Rs } 2146323 \\
A>C\end{array}$ & $\begin{array}{l}\text { Hautam'a'ki } \\
(2013)\end{array}$ & Prospective & Finland & Caucasian & BVZ & 96 & 78.0 & 63.5 & 4 & 8 \\
\hline & & Hagstrom (2014) & Prospective & USA & Caucasian & $\begin{array}{l}\text { RBZ or } \\
\text { BVZ }\end{array}$ & 835 & 78.5 & 61.2 & 12 & 8 \\
\hline & Rs 1413711 & McKibbin (2012) & Prospective & UK & Caucasian & $\mathrm{RBZ}$ & 104 & 81.5 & 55.8 & 6 & 7 \\
\hline & $\mathrm{T}>\mathrm{C}$ & Hagstrom (2014) & Prospective & USA & Caucasian & $\begin{array}{l}\text { RBZ or } \\
\text { BVZ }\end{array}$ & 835 & 78.5 & 61.2 & 12 & 8 \\
\hline & $\begin{array}{l}\text { Rs } 2010963 \\
\text { C>G }\end{array}$ & Kitchens (2013) & Retrospective & USA & Caucasian & $\begin{array}{l}\text { RBZ or } \\
\text { BVZ }\end{array}$ & 99 & 80.0 & 67.3 & 4 & 8 \\
\hline & & Hagstrom (2014) & Prospective & USA & Caucasian & $\begin{array}{l}\text { RBZ or } \\
\text { BVZ }\end{array}$ & 835 & 78.5 & 61.2 & 12 & 8 \\
\hline & $\begin{array}{l}\text { Rs1570360 } \\
G>A\end{array}$ & Kitchens (2013) & Retrospective & USA & Caucasian & $\begin{array}{l}\text { RBZ or } \\
\text { BVZ }\end{array}$ & 99 & 80.0 & 67.3 & 4 & 8 \\
\hline & & Lazzeri (2013) & Prospective & Italy & Caucasian & $\mathrm{RBZ}$ & 64 & 76.3 & 45.3 & 9 & 7 \\
\hline \multirow[t]{2}{*}{ VEGFR2 } & $\begin{array}{l}\text { Rs } 2071559 \\
A>G\end{array}$ & Hagstrom (2014) & Prospective & USA & Caucasian & $\begin{array}{l}\text { RBZ or } \\
\text { BVZ }\end{array}$ & 835 & 78.5 & 61.2 & 12 & 8 \\
\hline & & $\begin{array}{l}\text { Cruz-Gonzalez } \\
(2014)\end{array}$ & Prospective & Spain & Caucasian & RBZ & 74 & 71.6 & 52.1 & 12 & 8 \\
\hline
\end{tabular}


article usually offered genotype data needed for several SNPs concurrently. Just as in Hagstrom et al's paper, ${ }^{20}$ we found information for rs699947, rs699946, rs833069, rs2146323, rs1413711 and rs2010963. The genotype distributions of each SNP are summarised in table 2.

\section{Genotype contrast}

We meta-analysed eight variations in VEGF-A, including rs699947, rs699946, rs833069, rs833061, rs2146323, rs1413711, rs2010963, rs1570360 and one variation rs2071559 in VEGFR-2. For each variation, we calculated a pooled OR based on genotype contrast. The results of meta-analysis are summarised in table 3 . Unexpectedly, there was only one SNP, rs833061, which showed a marginally significant association with response to treatment with anti-VEGF agents. For this polymorphism, anti-VEGF treatment was much more effective in patients with AMD having the CC genotype (CC vs TT: OR=2.222, 95\% CI 1.252 to $3.944, p=0.006$; CT vs TT: $\mathrm{OR}=2.537,95 \%$ CI 1.478 to $4.356, \mathrm{p}=0.001$ and CC vs $\mathrm{CT}+\mathrm{TT}$ : $\mathrm{OR}=2.362$, 95\% CI 1.414 to $3.946, \mathrm{p}=0.001$, respectively). However, the allele model ( $\mathrm{C}$ vs $\mathrm{T}$ ) and dominant model (CC+CT vs TT) were not associated with altered treatment response (C vs T: $\mathrm{OR}=1.26695 \% \mathrm{CI} 0.983$ to 1.631 , $\mathrm{p}=0.067$; CC+CT vs TT: $\mathrm{OR}=1.029,95 \%$ CI 0.718 to 1.476 , $\mathrm{p}=0.876$ ).

\section{Genetic model}

In this analysis, as for rs833061, the presence of CC versus TT genotypes and CT versus TT genotypes had a significant effect on the improved outcome as a result of anti-VEGF therapy ( $p=0.006$ and $p=0.001$, respectively), while the presence of the allele $T$ versus $C$ did not have a significant effect $(p=0.067)$. The genetic model indicating mode of inheritance is likely to be the dominant model $(\mathrm{CC}+\mathrm{CT}$ vs TT), whereas our analysis turned out not to be $(\mathrm{p}=0.876)$. On the contrary, in the contrasts of the CC versus CT+TT model, the patients with the CC genotype appeared to be associated with an elevated response to anti-VEGF treatment for nAMD (OR $=2.362,95 \%$ CI 1.414 to $3.946, p=0.001)$. In all of these comparisons, homogeneity was identified and, thus, fixed-effects models were applied to the data. In the subgroup analysis, rs833061 polymorphism was more likely to be a predictor of anti-VEGF treatment response for East Asians (CC vs TT: OR=2.903, 95\% CI 1.150 to $7.330, \mathrm{p}=0.024$; $\mathrm{CT}$ vs TT: $\mathrm{OR}=3.849,95 \% \mathrm{CI}$ 1.522 to $9.733, p=0.004$; and $\mathrm{CC}$ vs $\mathrm{CT}+\mathrm{TT}$ : $\mathrm{OR}=3.339$, $95 \%$ CI 1.369 to $8.145, p=0.008$, respectively). Similar to the main analysis, homogeneity was identified in this analysis as well and a fixed-effects model was applied to the data (figure 2A). Begger's test and Egger's test indicated no statistically significant evidence of publication bias for overall studies in all five genetic models $(\mathrm{p}>0.05)$.

Table 2 Genotype distributions of polymorphisms in studies included in the current meta-analysis

\begin{tabular}{|c|c|c|c|c|c|c|c|c|c|}
\hline \multirow[b]{2}{*}{ Gene } & \multirow[b]{2}{*}{ Polymorphism } & \multirow[b]{2}{*}{ Study group (year) } & \multirow[b]{2}{*}{ Definition of a good response } & \multicolumn{3}{|c|}{$\begin{array}{l}\text { No. of patients with good } \\
\text { response }(\%)\end{array}$} & \multicolumn{3}{|c|}{$\begin{array}{l}\text { Total no. of } \\
\text { patients }\end{array}$} \\
\hline & & & & $1^{*}$ & $2^{*}$ & $3^{*}$ & $1^{*}$ & $2^{*}$ & $3^{*}$ \\
\hline \multirow[t]{30}{*}{ VEGF-A } & Rs699947 & Hautam'a'ki (2013) & Retinal exudate resolved(measured by OCT) & $17(51.5)$ & 19 (40.4) & $8(50.0)$ & 33 & 47 & 16 \\
\hline & $A>C$ & Kitchens (2013) & Retinal exudate resolved(measured by OCT) & $6(24.0)$ & $11(20.4)$ & $3(15.0)$ & 25 & 54 & 20 \\
\hline & & Lazzeri (2013) & Retinal exudate resolved(measured by OCT) & $3(33.3)$ & $17(54.8)$ & $14(58.3)$ & 9 & 31 & 24 \\
\hline & & Park (2014) & Gain of $\geq 5$ letters & $21(75.0)$ & $50(42.4)$ & $62(50.0)$ & 28 & 118 & 124 \\
\hline & & Hagstrom (2014) & Gain of $\geq 5$ letters & $60(29.9)$ & $126(30.8)$ & 65 (28.9) & 201 & 409 & 225 \\
\hline & & & Retinal exudate resolved(measured by OCT) & 44 (21.9) & $137(33.5)$ & $58(25.8)$ & & & \\
\hline & & Cruz-Gonzalez (2014) & Gain of $\geq 5$ letters & $11(68.8)$ & $20(43.5)$ & $15(46.9)$ & 16 & 46 & 32 \\
\hline & & & Retinal exudate resolved(measured by OCT) & $12(75.0)$ & $30(65.2)$ & $18(56.3)$ & & & \\
\hline & & Van Asten (2014) & Loss of $\mathrm{VA}<30 \%$ of letters & 98 (29.6) & $158(47.7)$ & $75(22.7)$ & 113 & 179 & 83 \\
\hline & Rs 699946 & Park (2014) & Gain of $\geq 5$ letters & 45 (47.9) & $67(51.1)$ & $24(50.0)$ & 94 & 131 & 48 \\
\hline & $A>G$ & Hagstrom (2014) & Gain of $\geq 5$ letters & $167(31.2)$ & $78(29.0)$ & $6(19.4)$ & 535 & 269 & 31 \\
\hline & & & Retinal exudate resolved(measured by OCT) & $150(28.0)$ & $78(29.0)$ & $11(35.5)$ & & & \\
\hline & Rs 833069 & Hagstrom (2014) & Gain of $\geq 5$ letters & $29(28.2)$ & $111(30.9)$ & $111(29.8)$ & 103 & 359 & 373 \\
\hline & $C>T$ & & Retinal exudate resolved(measured by OCT) & $23(22.3)$ & $114(31.8)$ & $102(27.3)$ & & & \\
\hline & & Van Asten (2014) & Loss of $\mathrm{VA}<30 \%$ of letters & $45(13.8)$ & $137(42.2)$ & $143(44.0)$ & 52 & 158 & 158 \\
\hline & Rs 833061 & Kitchens (2013) & Retinal exudate resolved(measured by OCT) & $3(15.0)$ & $11(20.4)$ & $6(24.0)$ & 20 & 54 & 25 \\
\hline & $C>T$ & Park (2014) & Gain of $\geq 5$ letters & $21(75.0)$ & $53(43.8)$ & $62(50.8)$ & 28 & 121 & 122 \\
\hline & & Cruz-Gonzalez (2014) & Gain of $\geq 5$ letters & $14(70.0)$ & $19(41.3)$ & $13(50.0)$ & 20 & 46 & 28 \\
\hline & & & Retinal exudate resolved(measured by OCT) & $16(80.0)$ & $28(60.9)$ & $16(57.1)$ & & & \\
\hline & Rs 2146323 & Hautam'a'ki (2013) & Retinal exudate resolved(measured by OCT) & $8(57.1)$ & $20(43.5)$ & $16(44.4)$ & 14 & 46 & 36 \\
\hline & $A>C$ & Hagstrom (2014) & Gain of $\geq 5$ letters & $27(27.8)$ & $120(31.8)$ & $104(28.8)$ & 97 & 377 & 361 \\
\hline & & & Retinal exudate resolved(measured by OCT) & $23(23.7)$ & $113(30.0)$ & $103(28.5)$ & & & \\
\hline & Rs 1413711 & McKibbin (2012) & Gain of $\geq 5$ letters & $10(40.0)$ & $29(55.8)$ & $14(51.9)$ & 25 & 52 & 27 \\
\hline & $\mathrm{T}>\mathrm{C}$ & Hagstrom (2014) & Gain of $\geq 5$ letters & $62(30.0)$ & $122(30.1)$ & $67(29.1)$ & 207 & 398 & 230 \\
\hline & & & Retinal exudate resolved(measured by OCT) & $48(23.2)$ & $130(32.7)$ & $61(26.2)$ & & & \\
\hline & Rs 2010963 & Kitchens (2013) & Retinal exudate resolved(measured by OCT) & $9(20.0)$ & $10(23.3)$ & $1(9.0)$ & 45 & 43 & 11 \\
\hline & $\mathrm{C}>\mathrm{G}$ & Hagstrom (2014) & Gain of $\geq 5$ letters & $28(28.3)$ & $112(31.0)$ & $111(29.6)$ & 99 & 361 & 375 \\
\hline & & & Retinal exudate resolved(measured by OCT) & $23(23.2)$ & $112(31.0)$ & $104(27.7)$ & & & \\
\hline & Rs1570360 & Kitchens(2013) & Retinal exudate resolved(measured by OCT) & $1(8.3)$ & $9(20.0)$ & $10(23.8)$ & 12 & 45 & 42 \\
\hline & $A>G$ & Lazzeri (2013) & Retinal exudate resolved(measured by OCT) & $23(71.9)$ & $13(50.0)$ & $1(16.7)$ & 32 & 26 & 6 \\
\hline \multirow[t]{4}{*}{ VEGFR 2} & Rs 2071559 & Hagstrom (2014) & Gain of $\geq 5$ letters & $59(30.6)$ & $128(30.9)$ & $64(28.1)$ & 193 & 414 & 228 \\
\hline & $A>G$ & & Retinal exudate resolved(measured by OCT) & $58(30.0)$ & $108(26.0)$ & $73(32.0)$ & & & \\
\hline & & Cruz-Gonzalez (2014) & Gain of $\geq 5$ letters & $7(43.8)$ & $25(53.2)$ & $14(45.2)$ & 16 & 47 & 31 \\
\hline & & & Retinal exudate resolved(measured by OCT) & $10(62.5)$ & $33(70.2)$ & $17(54.8)$ & & & \\
\hline
\end{tabular}

Bold text highlights positive results.

$1 *$, Homozygous wild type; $2^{*}$, Heterozygous variant; $3^{*}$, Homozygous variant; OCT, optical coherence tomography; VA, visual acuity; VEGF-A, vascular endothelial growth factor A; VEGFR, vascular endothelial growth factor receptor. 
Table 3 Results of meta-analysis for polymorphisms and treatment response of nAMD

\begin{tabular}{|c|c|c|c|c|c|c|c|c|c|}
\hline \multirow[b]{2}{*}{ Gene } & \multirow[b]{2}{*}{ Polymorphism } & \multirow[b]{2}{*}{ No. of cohorts } & & \multirow[b]{2}{*}{ Genetic model } & \multicolumn{2}{|l|}{ FEM or REM* } & \multicolumn{2}{|c|}{ Heterogeneity } & \multirow[b]{2}{*}{ Egger's test $(p)$} \\
\hline & & & & & OR $(95 \% \mathrm{Cl})$ & $p$ & $P(Q)$ & $\mathrm{I}^{2}(\%)$ & \\
\hline \multirow[t]{70}{*}{ VEGF-A } & Rs699947 & Overall & & A vs $C$ & 1.030 (0.915 to 1.161$)$ & 0.622 & 0.370 & 7.8 & 0.282 \\
\hline & $A>C$ & & & $\mathrm{AA}$ vs $\mathrm{CC}$ & $1.078(0.842$ to 1.378$)$ & 0.553 & 0.133 & 35.6 & 0.349 \\
\hline & & & & $\mathrm{AC}$ vs $\mathrm{CC}$ & $1.089(0.894$ to 1.326$)$ & 0.156 & 0.559 & 0.0 & 0.161 \\
\hline & & & & $A A$ vs $A C+C C$ & 1.165 (0.787 to 1.726$)$ & 0.445 & 0.010 & 60.4 & 0.161 \\
\hline & & & & $A A+A C$ vs $C C$ & 1.083 (0.898 to 1.306$)$ & 0.403 & 0.887 & 0.0 & 0.281 \\
\hline & & Sub-analysis by ethnicity & Caucasian & A vs $C$ & 1.008 (0.888 to 1.143$)$ & 0.906 & 0.372 & 7.5 & n.a \\
\hline & & & & $A A$ vs $C C$ & $0.983(0.759$ to 1.273$)$ & 0.897 & 0.407 & 3.0 & n.a \\
\hline & & & & $\mathrm{AC}$ vs $\mathrm{CC}$ & 1.168 (0.942 to 1.448$)$ & 0.156 & 0.768 & 0.0 & n.a \\
\hline & & & & $A A$ vs $A C+C C$ & $0.983(0.707$ to 1.368$)$ & 0.930 & 0.105 & 41.0 & n.a \\
\hline & & & & $A A+A C$ vs $C C$ & 1.110 (0.905 to 1.360$)$ & 0.316 & 0.857 & 0.0 & n.a \\
\hline & & & East Asia & A vs $C$ & 1.238 (0.862 to 1.777$)$ & 0.247 & n.a & n.a & n.a \\
\hline & & & & $A A$ vs $C C$ & 3.000 (1.190 to 7.566$)$ & 0.020 & n.a & n.a & n.a \\
\hline & & & & $A C$ vs $C C$ & 0.735 (0.443 to 1.221$)$ & 0.235 & n.a & n.a & n.a \\
\hline & & & & $A A$ vs $A C+C C$ & 3.482 (1.427 to 8.496$)$ & 0.006 & n.a & n.a & n.a \\
\hline & & & & $\mathrm{AA}+\mathrm{AC}$ vs $\mathrm{CC}$ & 0.947 (0.586 to 1.528$)$ & 0.822 & n.a & n.a & n.a \\
\hline & Rs 699946 & Overall & & A vs $\mathrm{G}$ & 1.016 (0.863 to 1.198$)$ & 0.846 & 0.332 & 9.3 & n.a \\
\hline & $A>G$ & & & $A A$ vs $G G$ & 1.031 (0.665 to 1.600$)$ & 0.890 & 0.253 & 27.3 & 0.841 \\
\hline & & & & $A G$ vs $G G$ & 1.066 (0.687 to 1.654$)$ & 0.776 & 0.407 & 0.0 & 0.408 \\
\hline & & & & $\mathrm{AA}$ vs $\mathrm{AG}+\mathrm{GG}$ & 1.013 (0.828 to 1.239$)$ & 0.900 & 0.497 & 0.0 & 0.657 \\
\hline & & & & $A A+A G$ vs $G G$ & 1.051 (0.692 to 1.597$)$ & 0.815 & 0.294 & 18.2 & 0.685 \\
\hline & & Sub-analysis by ethnicity & Caucasian & $A$ vs $G$ & $1.039(0.862$ to 1.254$)$ & 0.686 & 0.159 & 49.5 & n.a \\
\hline & & & & $A A$ vs $G G$ & 1.114 (0.629 to 1.927$)$ & 0.712 & 0.103 & 62.5 & n.a \\
\hline & & & & $A G$ vs $G G$ & 1.081 (0.600 to 1.947$)$ & 0.795 & 0.179 & 44.5 & n.a \\
\hline & & & & $A A$ vs $A G+G G$ & 1.039 (0.834 to 1.295$)$ & 0.733 & 0.298 & 7.6 & n.a \\
\hline & & & & $A A+A G$ vs $G G$ & 1.103 (0.625 to 1.944$)$ & 0.735 & 0.119 & 58.9 & n.a \\
\hline & & & East Asia & A vs $G$ & 0.944 (0.672 to 1.327$)$ & 0.739 & n.a & n.a & n.a \\
\hline & & & & $A A$ vs $\mathrm{GG}$ & 0.918 (0.458 to 1.841$)$ & 0.810 & n.a & n.a & n.a \\
\hline & & & & $A G$ vs $G G$ & 1.047 (0.540 to 2.028$)$ & 0.892 & n.a & n.a & n.a \\
\hline & & & & $\mathrm{AA}$ vs $\mathrm{AG}+\mathrm{GG}$ & 0.888 (0.539 to 1.464$)$ & 0.642 & n.a & n.a & n.a \\
\hline & & & & $A A+A G$ vs $G G$ & 0.991 (0.531 to 1.848$)$ & 0.978 & n.a & n.a & n.a \\
\hline & Rs 833069 & Caucasian & & C vs T & 0.961 (0.827 to 1.115$)$ & 0.598 & 0.650 & 0.0 & 0.514 \\
\hline & $C>T$ & & & CC vs TT & 0.824 (0.591 to 1.148$)$ & 0.253 & 0.790 & 0.0 & 0.306 \\
\hline & & & & CT vs TT & $1.088(0.880$ to 1.347$)$ & 0.435 & 0.313 & 14.0 & 0.306 \\
\hline & & & & $\mathrm{CC}$ vs $\mathrm{CT}+\mathrm{TT}$ & 0.795 (0.582 to 1.085$)$ & 0.149 & 0.728 & 0.0 & 0.496 \\
\hline & & & & $\mathrm{CC}+\mathrm{CT}$ vs $\mathrm{TT}$ & $1.026(0.839$ to 1.255$)$ & 0.803 & 0.412 & 0.0 & 0.996 \\
\hline & Rs 833061 & Overall & & C vs T & 1.266 (0.983 to 1.631$)$ & 0.067 & 0.406 & 0.0 & 0.882 \\
\hline & $C>T$ & & & CC vs TT & 2.222 (1.252 to 3.944$)$ & 0.006 & 0.292 & 19.6 & 0.326 \\
\hline & & & & CT vs TT & 2.537 (1.478 to 4.356$)$ & 0.001 & 0.227 & 30.9 & 0.882 \\
\hline & & & & $\mathrm{CC}$ vs $\mathrm{CT}+\mathrm{TT}$ & 2.362 (1.414 to 3.946$)$ & 0.001 & 0.214 & 33.1 & 0.145 \\
\hline & & & & $\mathrm{CC}+\mathrm{CT}$ vs $\mathrm{TT}$ & 1.029 (0.718 to 1.476$)$ & 0.876 & 0.758 & 0.0 & 0.765 \\
\hline & & Sub-analysis by ethnicity & Caucasian & C vs T & $1.302(0.912$ to 1.859$)$ & 0.146 & 0.239 & 30.1 & n.a \\
\hline & & & & CC vs TT & 1.845 (0.882 to 3.861$)$ & 0.104 & 0.200 & 37.9 & n.a \\
\hline & & & & CT vs TT & 1.989 (1.011 to 3.910$)$ & 0.046 & 0.204 & 37.0 & n.a \\
\hline & & & & $\mathrm{CC}$ vs $\mathrm{CT}+\mathrm{TT}$ & $1.937(1.025$ to 3.661$)$ & 0.042 & 0.163 & 44.9 & n.a \\
\hline & & & & $\mathrm{CC}+\mathrm{CT}$ vs $\mathrm{TT}$ & 1.136 (0.655 to 1.969$)$ & 0.649 & 0.618 & 0.0 & n.a \\
\hline & & & East Asia & C vs T & 1.231 (0.859 to 1.763$)$ & 0.258 & n.a & n.a & n.a \\
\hline & & & & CC vs TT & $2.903(1.150$ to 7.330$)$ & 0.024 & n.a & n.a & n.a \\
\hline & & & & CT vs TT & 3.849 (1.522 to 9.733$)$ & 0.004 & n.a & n.a & n.a \\
\hline & & & & $\mathrm{CC}$ vs $\mathrm{CT}+\mathrm{TT}$ & 3.339 (1.369 to 8.145$)$ & 0.008 & n.a & n.a & n.a \\
\hline & & & & $\mathrm{CC}+\mathrm{CT}$ vs TT & 0.955 (0.592 to 1.541$)$ & 0.850 & n.a & n.a & n.a \\
\hline & Rs 2146323 & Caucasian & & A vs $C$ & 1.000 (0.859 to 1.164$)$ & 1.000 & 0.701 & 0.0 & 0.534 \\
\hline & $A>C$ & & & AA vs CC & 0.909 (0.644 to 1.282$)$ & 0.585 & 0.526 & 0.0 & 0.465 \\
\hline & & & & $A C$ vs $C C$ & $1.103(0.888$ to 1.369$)$ & 0.375 & 0.903 & 0.0 & 0.356 \\
\hline & & & & $A A$ vs $A C+C C$ & 0.867 (0.627 to 1.199$)$ & 0.389 & 0.434 & 0.0 & 0.500 \\
\hline & & & & $\mathrm{AA}+\mathrm{AC}$ vs $\mathrm{CC}$ & 1.061 (0.863 to 1.304$)$ & 0.574 & 0.902 & 0.0 & 0.277 \\
\hline & Rs 1413711 & Caucasian & & T vs $C$ & 0.961 (0.832 to 1.109$)$ & 0.583 & 0.635 & 0.0 & n.a \\
\hline & $\mathrm{T}>\mathrm{C}$ & & & TT vs CC & $0.912(0.683$ to 1.216$)$ & 0.530 & 0.600 & 0.0 & 0.456 \\
\hline & & & & TC vs CC & $1.199(0.940$ to 1.531$)$ & 0.144 & 0.688 & 0.0 & 0.456 \\
\hline & & & & TT vs TC+CC & 0.811 (0.638 to 1.032$)$ & 0.089 & 0.256 & 26.7 & 0.972 \\
\hline & & & & $\mathrm{TT}+\mathrm{TC}$ vs CC & 1.096 (0.871 to 1.379$)$ & 0.434 & 0.896 & 0.0 & 0.613 \\
\hline & Rs 2010963 & Caucasian & & C vs $\mathrm{G}$ & $0.994(0.852$ to 1.161$)$ & 0.943 & 0.892 & 0.0 & 0.229 \\
\hline & $\mathrm{C}>\mathrm{G}$ & & & CC vs GG & $0.722(0.340$ to 1.535$)$ & 0.398 & 0.040 & 68.9 & 0.242 \\
\hline & & & & CG vs GG & 0.874 (0.520 to 1.470$)$ & 0.612 & 0.025 & 72.9 & 0.871 \\
\hline & & & & CC vs CG+GG & 0.831 (0.604 to 1.144$)$ & 0.257 & 0.773 & 0.0 & 0.749 \\
\hline & & & & $\mathrm{CC}+\mathrm{CG}$ vs $\mathrm{GG}$ & $1.075(0.871$ to 1.327$)$ & 0.501 & 0.668 & 0.0 & 0.416 \\
\hline & Rs1570360 & Caucasian & & $A$ vs $G$ & $1.378(0.318$ to 5.973$)$ & 0.668 & 0.007 & 86.1 & n.a \\
\hline & $A>G$ & & & $A A$ vs $G G$ & 1.895 (0.046 to 77.497$)$ & 0.736 & 0.018 & 82.0 & n.a \\
\hline & & & & $A G$ vs $G G$ & 1.494 (0.269 to 8.295$)$ & 0.646 & 0.148 & 52.3 & n.a \\
\hline & & & & $A A$ vs $A G+G G$ & 1.241 (0.127 to 12.115$)$ & 0.852 & 0.050 & 74.0 & n.a \\
\hline & & & & $A A+A G$ vs $G G$ & 1.932 (0.169 to 22.029$)$ & 0.596 & 0.042 & 75.9 & n.a \\
\hline
\end{tabular}


Table 3 Continued

\begin{tabular}{|c|c|c|c|c|c|c|c|c|}
\hline \multirow[b]{2}{*}{ Gene } & \multirow[b]{2}{*}{ Polymorphism } & \multirow[b]{2}{*}{ No. of cohorts } & \multirow[b]{2}{*}{ Genetic model } & \multicolumn{2}{|l|}{ FEM or REM* } & \multicolumn{2}{|c|}{ Heterogeneity } & \multirow[b]{2}{*}{ Egger's test ( $p$ ) } \\
\hline & & & & OR $(95 \% \mathrm{Cl})$ & $\mathrm{p}$ & $P(Q)$ & $I^{2}(\%)$ & \\
\hline \multirow{5}{*}{ VEGFR 2} & Rs 2071559 & Caucasian & A vs $\mathrm{G}$ & $1.018(0.885$ to 1.171$)$ & 0.805 & 0.750 & 0.0 & 0.475 \\
\hline & $A>G$ & & $A A$ vs $G G$ & 1.025 (0.775 to 1.355$)$ & 0.862 & 0.865 & 0.0 & 0.475 \\
\hline & & & $A G$ vs $G G$ & $0.998(0.790$ to 1.261$)$ & 0.985 & 0.139 & 45.4 & 0.699 \\
\hline & & & $A A$ vs $A G+G G$ & $1.040(0.821$ to 1.318$)$ & 0.744 & 0.941 & 0.0 & 0.319 \\
\hline & & & $A A+A G$ vs $G G$ & $1.010(0.810$ to 1.258$)$ & 0.933 & 0.239 & 28.9 & 0.156 \\
\hline
\end{tabular}

Bold text highlights positive results.

*If the $p$ value for $Q$-statistic was $>0.10$ or the $I^{2}$ value was $>50 \%$, a REM was used, otherwise a FEM was adopted.

FEM, fixed-effects model; n.a, not available; nAMD, neovascular age-related macular degeneration; REM, random-effects model; VEGF-A, vascular endothelial growth factor A; VEGFR, vascular endothelial growth factor receptor.

The other eight variations did not show a significant association with results of anti-VEGF therapy in any inheritance models ( $p>0.05$; table 2$)$. Among the insignificant polymorphisms, rs2146323 $\left(\mathrm{I}^{2}=0.0 \%\right)$ showed no heterogeneity among studies, while rs1413711( $\left.\mathrm{I}^{2} \leq 26.7 \%\right)$, $\operatorname{rs} 699947\left(\mathrm{I}^{2} \leq 46.2 \%\right)$, rs699946 $\left(\mathrm{I}^{2} \leq 27.3 \%\right), \quad$ rs833069 $\quad\left(\mathrm{I}^{2} \leq 14.0 \%\right)$, rs 2010963 $\left(\mathrm{I}^{2} \leq 72.9 \%\right)$, rs1570360 $\left(\mathrm{I}^{2} \leq 86.1 \%\right)$ and $\mathrm{rs} 2071559\left(\mathrm{I}^{2} \leq 45.4 \%\right)$ showed moderate-to-high heterogeneities (table 2). To explain the heterogeneity, we performed subgroup analysis by ethnicity (see online Supplement part 1). Due to the limited number of studies, we only tested the associations of two SNPs, rs699947 and rs699946. Even though five genetic models of rs699947 were not associated with altered treatment response, when we divided the patients according to ethnicity (Caucasians vs East Asians), AA genotype was associated with an increased response to treatment of nAMD in Asians (AA vs TT: $\mathrm{OR}=3.000,95 \%$ CI 1.190 to $7.566, \mathrm{p}=0.020 ; \mathrm{AA}$ vs $\mathrm{AC}+\mathrm{CC}$ : $\mathrm{OR}=3.482,95 \%$ CI 1.427 to $8.496, \mathrm{p}=0.006$, respectively) but not in Caucasians (AA vs TT: OR $=0.983,95 \%$ CI 0.759 to 1.273 , $\mathrm{p}=0.897 ; \mathrm{AA}$ vs $\mathrm{AC}+\mathrm{CC}: \mathrm{OR}=0.983,95 \%$ CI 0.707 to 1.368 , $\mathrm{p}=0.930$ ). For the other rs699946, it still did not show any significant association between genotype and treatment whatever for Caucasians or Asians ( $p>0.05$ for both of the two groups) (table 2).
A

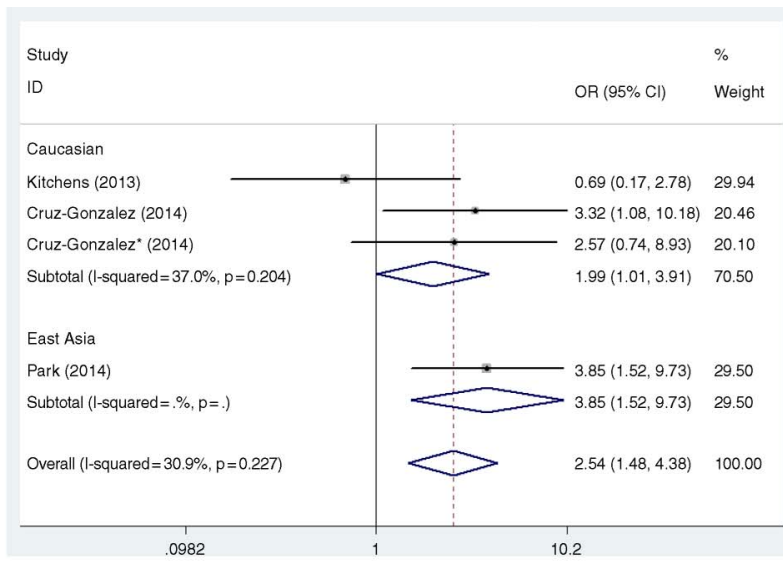

B

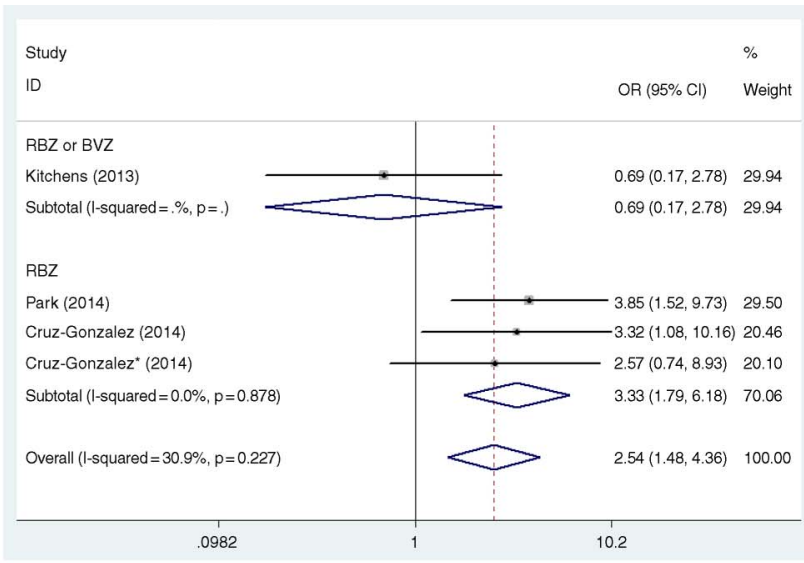

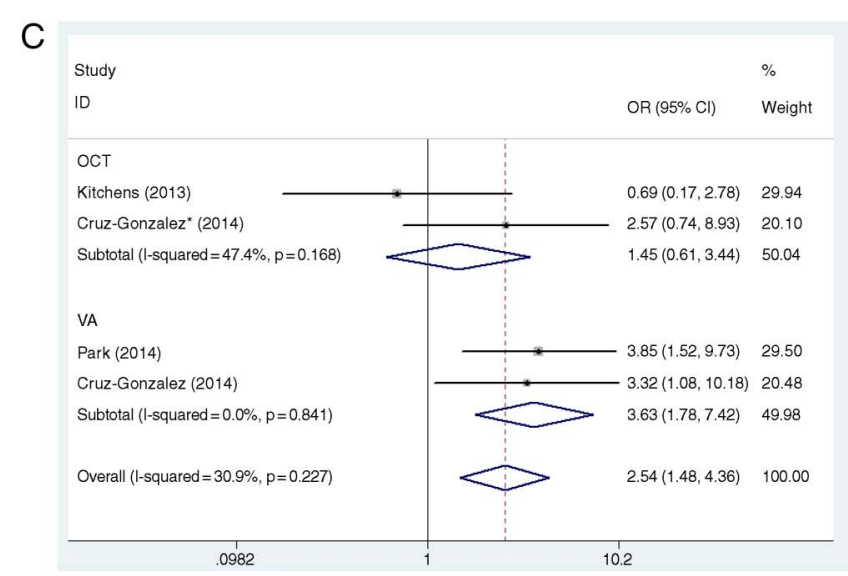

Figure 2 The association between VEGF-A polymorphism rs833061 and treatment response of neovascular age-related macular degeneration (nAMD) based on the heterozygote model (CT vs TT). (A) Sub-analysis by ethnicity, (B) sub-analysis by treatment and (C) sub-analysis by definition of a good response (DGR). 


\section{Sub-analysis}

From the genotype contrast and genetic model comparison, our analysis found that rs833061 may be a genetic predictor of response to treatment of nAMD with anti-VEGF agents. For all the trials included in this meta-analysis, some inevitable disadvantages included the heterogeneity of the treatment adopted (RBZ only, BVZ only, RBZ or BVZ), the criteria of positive treatment outcome (OCT or VA), the period for follow-up and so forth. To overcome the problem of heterogeneity in the treatment adopted and outcome definition, we decided to conduct a sub-analysis of studies that received RBZ treatment only or define a positive outcome only as improvement in VA.

\section{(1) Effect on treatment RBZ only}

In the limited studies for rs833061, a total of 459 patients were selected to receive RBZ treatment only. The results of this subanalysis revealed a stronger relationship between the presence of CT genotype and a positive outcome after anti-VEGF therapy. Thus, in the comparison of CT versus TT genotype, the OR increased from 2.537 (when all studies were included, table 3) to 3.327 (95\% CI 1.709 to $5.590, \mathrm{p}=0.000)$; similarly, in the comparison of CC versus CT+TT, OR increased from 2.362 to 3.091 (95\% CI 1.025 to $3.661, \mathrm{p}=0.000$ ), while the comparison between CC and TT remained practically unchanged at $\mathrm{OR}=2.222(95 \%$ CI 1.252 to $3.944, \mathrm{p}=0.001)$, with the statistical significance increased substantially from $\mathrm{p}=0.006$ to $\mathrm{p}=0.001$ (table 4 , figure 2B).

\section{(2) Effect on improvement of VA}

When we ran a sub-analysis for studies defining a good response as improvement in VA, results showed a stronger association between the CT genotype and a positive VA outcome after treatment. For the heterozygote model (CT vs TT), the OR increased from 2.537 to 3.631 (95\% CI 1.777 to 7.418 , $\mathrm{p}=0.000)$; equally, for the recessive model (CC vs CT+TT) and homozygote model (CC vs TT), the ORs elevated from 2.362 to 3.226 (95\% CI 1.630 to $6.385, \mathrm{p}=0.001)$ and from 2.222 to 2.827 (95\% CI 1.355 to $5.900, \mathrm{p}=0.006$ ), respectively (table 4 , figure $2 \mathrm{C})$.

\section{Assessment of potential biases and sensitivity analysis}

Like any other clinical research, original articles included in our meta-analysis have diverse clinical characteristics, with subtle differences in their inclusion criteria, anti-VEGF agents adopted, duration of follow-up, standardised assessment of outcome and so forth. To avoid possible selection bias, performance bias and detection bias for SNPs reported in four or more studies (ie, VEGF-A rs699947 and rs833061, VEGFR-2 rs2071559), sensitivity analyses were performed by sequentially omitting one study at a time (see online supplementary part 2, figure 3 ). The insignificant associations remained unchanged $(p>0.05$; data not shown). In the quality assessment of studies using the NOS, all of the studies were assigned $\geq 7$ points, indicating low risk of introducing biases. Therefore, no study was excluded from the meta-analysis due to poor quality. There was no significant publication bias detected by the funnel plots (see online Supplement part 3) and Egger's test (table 3 and figure 4).

\section{DISCUSSION}

Pharmacogenetics examines the impact of genetic variation on the response to drugs. It has been suggested that genetic factors may influence response to anti-VEGF treatment in nAMD. ${ }^{24} 25$ Several studies suggest that genetic variations in VEGF-A and VEGFR-2 may play a role in the pathogenesis; ${ }^{26-28}$ however, others have shown no association. ${ }^{29}{ }^{30}$ Genetic variants in the $V E G F-A$ and VEGFR-2 have been investigated in small-scale studies for their influence on anti-VEGF treatment outcomes with different conclusions. This study has, for the first time, summarised the associations of VEGF-related genes with response to anti-VEGF therapy. For the two most susceptible

Table 4 Results of sub-analysis by treatment or DGR for the VEGF-A rs833061 polymorphism and treatment response of nAMD

\begin{tabular}{|c|c|c|c|c|c|}
\hline \multirow[b]{2}{*}{ No. of cohorts } & \multirow[b]{2}{*}{ Genetic model } & \multicolumn{2}{|l|}{ FEM or REM* } & \multicolumn{2}{|c|}{ Heterogeneity } \\
\hline & & OR $(95 \% \mathrm{Cl})$ & $\mathrm{p}(\mathrm{Q})$ & $\mathrm{p}(\mathrm{Q})$ & $I^{2}(\%)$ \\
\hline \multicolumn{6}{|c|}{ Sub-analysis by treatment } \\
\hline RBZ & $\begin{array}{l}\text { C vs T } \\
\text { CC vs TT } \\
\text { CT vs TT } \\
\text { CC vs CT+TT } \\
\text { CC+CT vs TT }\end{array}$ & $\begin{array}{l}1.365(1.039 \text { to } 1.792) \\
2.222(1.252 \text { to } 3.944) \\
3.327(1.709 \text { to } 5.590) \\
3.091 \text { (1.025 to } 3.661) \\
1.071 \text { (0.731 to } 1.568)\end{array}$ & $\begin{array}{l}0.025 \\
0.001 \\
0.000 \\
0.000 \\
0.726\end{array}$ & $\begin{array}{l}0.684 \\
0.992 \\
0.878 \\
0.965 \\
0.678\end{array}$ & $\begin{array}{l}0.0 \\
0.0 \\
0.0 \\
0.0 \\
0.0\end{array}$ \\
\hline RBZ or BVZ & $\begin{array}{l}\text { C vs T } \\
\text { CC vs TT } \\
\text { CT vs TT } \\
\text { CC vs CT+TT } \\
\text { CC+CT vs TT }\end{array}$ & $\begin{array}{l}0.778(0.386 \text { to } 1.566) \\
0.559(0.121 \text { to } 2.587) \\
0.690(0.171 \text { to } 2.782) \\
0.644(1.369 \text { to } 8.145) \\
0.739(0.249 \text { to } 2.190)\end{array}$ & $\begin{array}{l}0.481 \\
0.457 \\
0.602 \\
0.519 \\
0.585\end{array}$ & $\begin{array}{l}\text { n.a } \\
\text { n.a } \\
\text { n.a } \\
\text { n.a } \\
\text { n.a }\end{array}$ & $\begin{array}{l}\text { n.a } \\
\text { n.a } \\
\text { n.a } \\
\text { n.a } \\
\text { n.a }\end{array}$ \\
\hline \multicolumn{6}{|c|}{ Sub-analysis by DGR } \\
\hline VA & $\begin{array}{l}\text { C vs T } \\
\text { CC vs TT } \\
\text { CT vs TT } \\
\text { CC vs } \mathrm{CT}+\mathrm{TT} \\
\text { CC+CT vs TT }\end{array}$ & $\begin{array}{l}1.307(0.963 \text { to } 1.773) \\
2.827(1.355 \text { to } 5.900) \\
3.631 \text { (1.777 to } 7.418) \\
3.226(1.630 \text { to } 6.385) \\
0.997 \text { (0.654 to } 1.518)\end{array}$ & $\begin{array}{l}0.085 \\
0.006 \\
0.000 \\
0.001 \\
0.988\end{array}$ & $\begin{array}{l}0.534 \\
0.923 \\
0.841 \\
0.903 \\
0.712\end{array}$ & $\begin{array}{l}0.0 \\
0.0 \\
0.0 \\
0.0 \\
0.0\end{array}$ \\
\hline ОСТ & $\begin{array}{l}\text { C vs T } \\
\text { CC vs TT } \\
\text { CT vs TT } \\
\text { CC vs CT+TT } \\
\text { CC+CT vs TT }\end{array}$ & $\begin{array}{l}1.181 \text { (0.751 to } 1.857) \\
1.463(0.571 \text { to } 3.746) \\
1.446(0.607 \text { to } 3.441) \\
1.458 \text { (0.647 to } 3.285) \\
1.125 \text { (0.558 to } 2.269)\end{array}$ & $\begin{array}{l}0.472 \\
0.428 \\
0.405 \\
0.363 \\
0.742\end{array}$ & $\begin{array}{l}0.122 \\
0.104 \\
0.168 \\
0.114 \\
0.327\end{array}$ & $\begin{array}{l}58.2 \\
62.1 \\
47.4 \\
59.9 \\
0.0\end{array}$ \\
\hline
\end{tabular}

If the $p$ value for $Q$-statistic was $>0.10$ or the $I 2$ value was $\geq 50 \%$, a REM was used, otherwise a FEM was adopted.

BVZ, bevacizumab; DGR, definition of a good response; FEM, fixed-effects model; nAMD, neovascular age-related macular degeneration; OCT, optical coherence tomography; RBZ, ranibizumab; REM, random-effects model; VA, visual acuity; VEGF-A, vascular endothelial growth factor A. 
Figure 3 Sensitivity analysis for rs699947 (A vs C) is shown.
Meta-analysis estimates, given named study is omitted

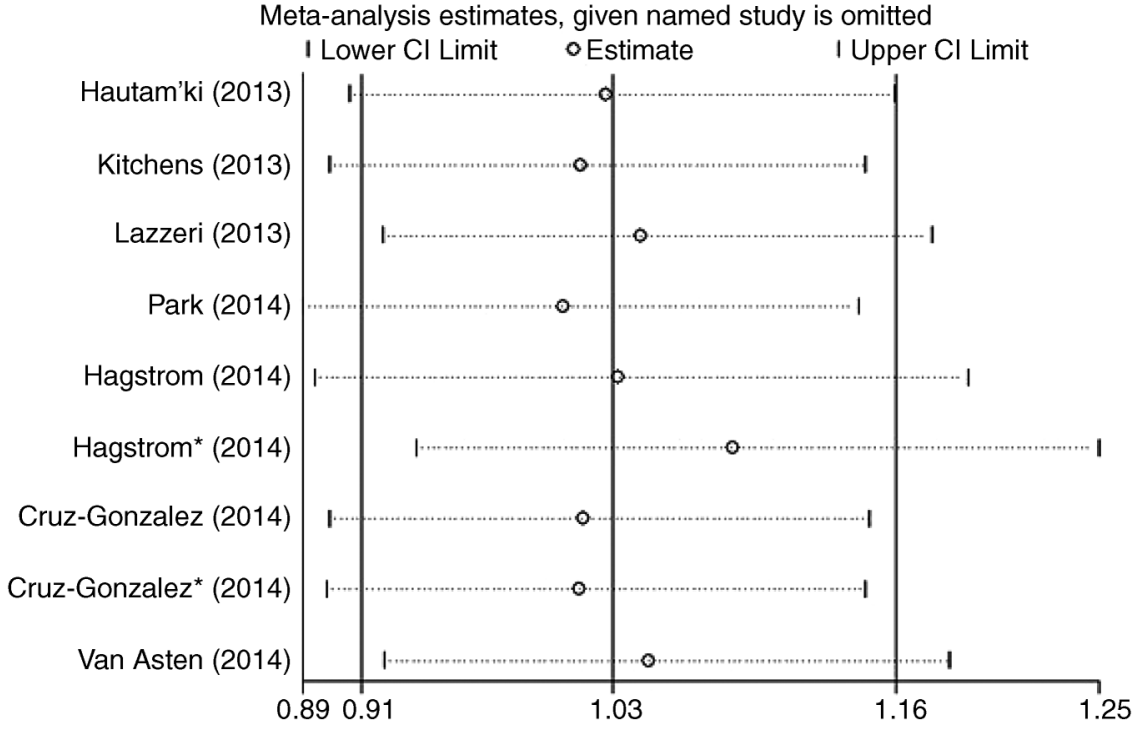

genes, VEGF-A and VEGFR-2, we performed meta-analyses on nine polymorphisms. Unexpectedly, we found only one SNP, rs833061 in VEGF-A, being marginally associated with response to anti-VEGF treatment.

The polymorphisms selected for this meta-analysis are genetic variants in VEGF-A and VEGFR-2 that are best known to be associated with clinical outcomes in VEGF-mediated diseases such as nAMD, diabetic retinopathy and several malignancies. ${ }^{31}$ Some of these SNPs are located in the promoter region and are known to influence the expression and plasma concentration of VEGF. $^{32}$ Others are located within the introns where there are putative regulatory elements influencing binding of VEGF to its receptor. ${ }^{33}$ One SNP is located in the promoter region of the gene encoding for VEGFR-2, the primary receptor responsible for the majority of the angiogenic effects of VEGF.

The five SNPs that we evaluated in the promoter region of VEGF- $A$ are rs833061, rs699946, rs699947, rs1570360 and rs2010963. These variants affect gene splicing, resulting in changes in VEGF expression levels. In our analysis, rs833061 showed a suggestive association with no to moderate heterogeneities in different genetic models. This SNP was reported in three studies. ${ }^{12} \quad 13 \quad 21$ Although a significant association was reported only in one study, ${ }^{16}$ for the recessive model, the effect of the genotype CC pointed to the same direction in studies of

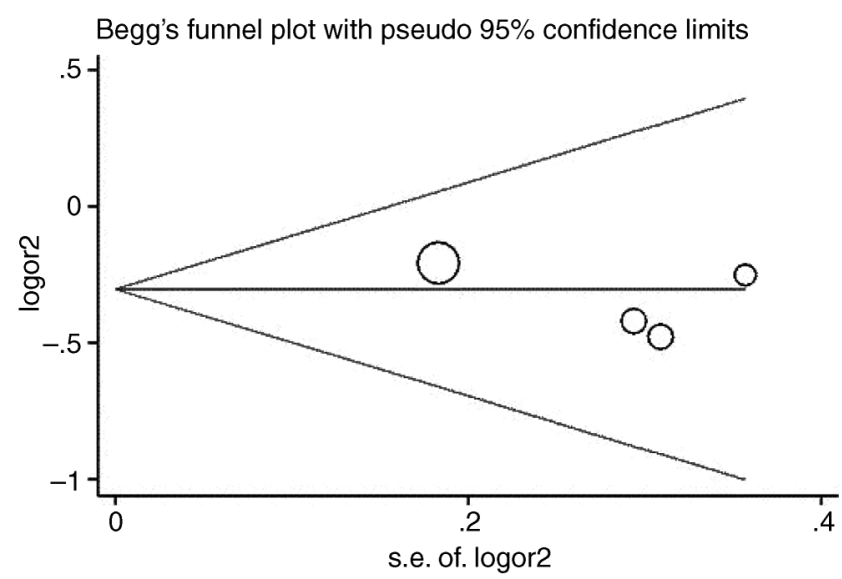

Figure 4 Begg's funnel plot with pseudo 95\% Cls for publication bias for rs833061 (CC vs TT) is shown.
Cruz-Gonzalez et $a l^{20}$ and Park et $a l^{12}\left(\mathrm{OR}=1.619^{16}\right.$ and $\mathrm{OR}=3.442,{ }^{12}$ with VA being the criterion), which strongly predicted a pooled $\mathrm{OR}=2.362$ for the total analysis and the result was statistically significant $(p=0.001)$, indicating that anti-VEGF treatment was more effective in patients with AMD having the rs833061 CC genotype. This relationship was strengthened further and became highly significant when a sub-analysis was conducted including studies with a VA-positive outcome criterion or the treatment of RBZ only, with $\mathrm{OR}=3.226$ or $\mathrm{OR}=3.091$, respectively. For this polymorphism, $\mathrm{C}$ allele has been demonstrated to be associated with increased promoter activity, ${ }^{29}$ which could be due to a lower expression of VEGF-A in patients expressing CC, which could encourage one to favour RBZ's antiangiogenic effect leading to a better response, which would explain the better response to the treatment. Another three insignificant SNPs (rs699946, rs2010963, rs1570360) lacked significant association in any of the studies with low-to-high heterogeneities. Nevertheless, even for these insignificant SNPs reported here, we could not completely deny their potential value acting as genetic predictors of anti-VEGF treatment for nAMD. Limited included articles restricted further statistical analysis and may lead to a robust result.

The three SNPs that we evaluated in the intron regions of $V E G F-A$ are rs1413711, rs2146323 and rs833069. The first of these, rs1413711, is located in intron 1 . It has been proposed that the proximity of this SNP to a putative stress response element-binding site may influence VEGF receptor binding and increase protein production. ${ }^{33}$ The remaining two intronic SNPs are located with intron 2. The variation rs2146323 (Caucasians) showed no association with response to anti-VEGF therapy in all of the tested populations $(p>0.05)$ with no heterogeneity $\left(\mathrm{I}^{2}=0.0 \%\right)$, indicating that it is not likely to be genetic markers for anti-VEGF therapy. Polymorphism rs833069 have been associated with the development and progression of $\mathrm{AMD},{ }^{27}$ although no significant pharmacogenetic effect has been found in our analysis.

Finally, rs2071559 is located in the gene that encodes the primary receptor responsible for the majority of the angiogenic effects of VEGF, the VEGFR-2 (kinase insert domain-containing receptor, KDR). A recent study found association between rs2071559 and AMD risk; this study suggests that the increase in KDR function is correlated with an increase in VEGF signalling, leading to pathological microvascular permeability, 
endothelial cell proliferation, invasion and migration and ultimately causing the neovascular lesions typical of AMD. ${ }^{27}$

This meta-analysis also reveals several limitations in the existing genetic studies of anti-VEGF therapy for nAMD. First, the small number of published genetic studies on VEGF-related genes in anti-VEGF therapy restricted the power of determining the associations, especially among different ethnic groups. Furthermore, the absence of any significant associations between SNPs in the VEGF pathway and response to anti-VEGF treatment contradicted the findings of several previous studies, many of which were limited by non-standardised assessment of outcomes and different anti-VEGF agents or regime or follow-up period adopted. Finally, as the pathogenesis of AMD is multifactorial, it would be more informative to test genetic, environmental (eg, smoking) factors and their interactions in the study population. Many efforts are underway to identify clinical, genetic and pharmacological biomarkers that could predict response to therapy, thereby providing important information to clinical decision-making and treatment options.

In conclusion, this is the first systematic review and meta-analysis focused on the association of VEGF-related genes with response to anti-VEGF therapy. Our analysis provides evidence that pharmacogenetics of VEGF-A polymorphisms rs833061 potentially plays a role in the frequency of the positive outcome in anti-VEGF treatment for nAMD, particularly among Asians, specifically when improvement in VA is taken as the standardised assessment of outcome or with RBZ only regime. Since the overall number of studies is small, it would be important to continue our study in order to confirm these results in a larger cohort and validate the possible predictive value of different polymorphisms for treatment response.

Acknowledgements The authors express their gratitude to all participants in this study.

Contributors Conceived and designed the experiments: WMX, XHB and ZXY. Performed the experiments: WMX, XXJ and ZHM. Analysed the data: WMX, ZMM and WXQ. Wrote the paper: WMX, XHB and XY. All authors read and approved the final manuscript.

Funding This work was supported by grants from the National Natural Science Foundation of China (No. 81170858, 81401423, 81501487).

Competing interests None declared.

Provenance and peer review Not commissioned; externally peer reviewed.

Open Access This is an Open Access article distributed in accordance with the Creative Commons Attribution Non Commercial (CC BY-NC 4.0) license, which permits others to distribute, remix, adapt, build upon this work non-commercially, and license their derivative works on different terms, provided the original work is properly cited and the use is non-commercial. See: http://creativecommons.org/ licenses/by-nc/4.0/

\section{REFERENCES}

1 Penn JS, Madan A, Caldwell RB, et al. Vascular endothelial growth factor in eye disease. Prog Retin Eye Res 2008;27:331-71.

2 Chen G, Li W, Tzekov R, et al. Bevacizumab versus ranibizumab for neovascular age-related macular degeneration: a meta-analysis of randomized controlled trials. Retina (Philadelphia, Pa) 2015:35:187-93.

3 Martin DF, Maguire MG. Ranibizumab and bevacizumab for treatment of neovascular age-related macular degeneration: two-year results. Ophthalmology 2012;119:1388-98.

4 Chakravarthy U, Harding SP, Rogers CA, et al. Ranibizumab versus bevacizumab to treat neovascular age-related macular degeneration: one-year findings from the IVAN randomized trial. Ophthalmology 2012;119:1399-411.

5 Krebs I, Schmetterer L, Boltz A, et al. A randomised double-masked trial comparing the visual outcome after treatment with ranibizumab or bevacizumab in patients with neovascular age-related macular degeneration. $\mathrm{Br} J$ Ophthalmol 2013;97:266-71

6 Priya RR, Chew EY, Swaroop A. Genetic studies of age-related macular degeneration: lessons, challenges, and opportunities for disease management. Ophthalmology 2012;119:2526-36.
7 Agosta E, Lazzeri S, Orlandi P, et al. Pharmacogenetics of antiangiogenic and antineovascular therapies of age-related macular degeneration. Pharmacogenomics 2012;13:1037-53.

8 Koch S, Claesson-Welsh L. Signal transduction by vascular endothelial growth factor receptors. Cold Spring Harb Perspect Med 2012;2:a006502.

9 Ferrara N, Damico L, Shams N, et al. Development of ranibizumab, an anti-vascular endothelial growth factor antigen binding fragment, as therapy for neovascular age-related macular degeneration. Retina (Philadelphia, Pa) 2006:26:859-70.

10 Huang C, Xu Y, Li X, et al. Vascular endothelial growth factor A polymorphisms and age-related macular degeneration: a systematic review and meta-analysis. $\mathrm{Mol}$ Vis 2013:19:1211-21.

11 Lazzeri S, Figus $M$, Orlandi $P$, et al. VEGF-A polymorphisms predict short-term functional response to intravitreal ranibizumab in exudative age-related macular degeneration. Pharmacogenomics 2013;14:623-30

12 Park UC, Shin JY, Kim SJ, et al. Genetic factors associated with response to intravitreal ranibizumab in Korean patients with neovascular age-related macular degeneration. Retina (Philadelphia, Pa) 2014;34:288-97.

13 Kitchens JW, Kassem N, Wood W, et al. A pharmacogenetics study to predict outcome in patients receiving anti-VEGF therapy in age related macular degeneration. Clin Ophthalmol 2013;7:1987-93.

14 Higgins JP, Thompson SG. Quantifying heterogeneity in a meta-analysis. Stat Med 2002;21:1539-58.

15 Higgins JP, Thompson SG, Deeks JJ, et al. Measuring inconsistency in meta-analyses. BMJ 2003:327:557-60.

16 Kuritz SJ, Landis JR, Koch GG. A general overview of Mantel-Haenszel methods: applications and recent developments. Annu Rev Public Health 1988;9:123-60.

17 Peters JL, Sutton AJ, Jones DR, et al. Contour-enhanced meta-analysis funnel plots help distinguish publication bias from other causes of asymmetry. J Clin Epidemiol 2008;61:991-6.

18 Sterne J A, Gavaghan D, Egger M. Publication and related bias in meta-analysis: power of statistical tests and prevalence in the literature. J Clin Epidemiol 2000:53:1119-29.

19 Hautamäki A, Kivioja J, Vavuli S, et al. Interleukin 8 promoter polymorphism predicts the initial response to bevacizumab treatment for exudative age-related macular degeneration. Retina (Philadelphia, Pa) 2013;33:1815-27.

20 Hagstrom SA, Ying GS, Pauer GJ, et al. VEGFA and VEGFR2 gene polymorphisms and response to anti-vascular endothelial growth factor therapy: comparison of age-related macular degeneration treatments trials (CATT). JAMA Ophthalmol 2014;132:521-7

21 Cruz-Gonzalez F, Cabrillo-Estévez L, López-Valverde G, et al. Predictive value of VEGF $A$ and VEGFR2 polymorphisms in the response to intravitreal ranibizumab treatment for wet AMD. Graefes Arch Clin Exp Ophthalmol 2014;252:469-75.

22 van Asten F, Rovers MM, Lechanteur YT, et al. Predicting non-response to ranibizumab in patients with neovascular age-related macular degeneration. Ophthalmic Epidemiol 2014:21:347-55.

23 McKibbin M, Ali M, Bansal S, et al. CFH, VEGF and HTRA1 promoter genotype may influence the response to intravitreal ranibizumab therapy for neovascular age-related macular degeneration. Br J Ophthalmol 2012;96:208-12.

24 Kanoff J, Miller J. Pharmacogenetics of the treatment response of age-related macular degeneration with ranibizumab and bevacizumab. Semin Ophthalmol 2013;28:355-60.

25 Fauser S, Lambrou GN. Genetic predictive biomarkers of anti-VEGF treatment response in patients with neovascular age-related macular degeneration. Surv Ophthalmol 2015:60:138-52.

26 Almeida LN, Melilo-Carolino R, Veloso CE, et al. Homozygosity for the $+674 C>T$ polymorphism on VEGF gene is associated with age-related macular degeneration in a Brazilian cohort. Graefes Arch Clin Exp Ophthalmol 2012;250:185-9.

27 Galan A, Ferlin A, Caretti L, et al. Association of age-related macular degeneration with polymorphisms in vascular endothelial growth factor and its receptor. Ophthalmology 2010;117:1769-74.

28 Lazzeri S, Orlandi P, Figus M, et al. The rs2071559 AA VEGFR-2 genotype frequency is significantly lower in neovascular age-related macular degeneration patients. ScientificWorldJournal 2012;2012:420190.

29 Janik-Papis K, Zaras M, Krzyzanowska A, et al. Association between vascular endothelial growth factor gene polymorphisms and age-related macular degeneration in a Polish population. Exp Mol Pathol 2009;87:234-8.

30 Fang AM, Lee AY, Kulkarni $M$, et al. Polymorphisms in the VEGFA and VEGFR-2 genes and neovascular age-related macular degeneration. Mol Vis 2009:15:2710-19.

31 Pasqualetti G, Danesi R, Del Tacca $M$, et al. Vascular endothelial growth factor pharmacogenetics: a new perspective for anti-angiogenic therapy. Pharmacogenomics 2007:8:49-66.

32 Harper SJ, Bates DO. VEGF-A splicing: the key to anti-angiogenic therapeutics? Nat Rev Cancer 2008;8:880-7.

33 Churchill AJ, Carter JG, Lovell HC, et al. VEGF polymorphisms are associated with neovascular age-related macular degeneration. Hum Mol Genet 2006;15: 2955-61. 